\title{
Um estudo correlacional entre habilidades sociais e traços de personalidade
}

\author{
José Maurício Haas Bueno ${ }^{1}$ \\ Sandra Maria da Silva Sales Oliveira \\ José Carlos da Silva Oliveira
}

\begin{abstract}
Resumo
Este trabalho teve como objetivo o desenvolvimento de um estudo correlacional entre habilidades sociais e traços de personalidade segundo o modelo dos cinco grandes fatores. Foram sujeitos da pesquisa 189 estudantes universitários, de ambos os sexos (41 homens e 148 mulheres), de 18 a 59 anos (média 26,3 anos), primeiro-anistas dos cursos de Letras, História, Psicologia, Pedagogia e Biologia. Foram aplicados o Inventário de Habilidades Sociais, que informa sobre (a) enfrentamento com risco, (b) auto-afirmação na expressão de afeto positivo, (c) conversação e desenvoltura social, (d) autoexposição a desconhecidos e a situações novas e (e) auto-controle da agressividade a situações aversivas; e um Inventário de Personalidade que informa sobre os cinco grandes fatores: extroversão, socialização, escrupulosidade, neuroticismo e abertura para novas experiências. Encontrou-se que a variância de enfrentamento com risco pode ser explicada principalmente por extroversão e abertura para novas experiências; auto-afirmação na expressão de afetos positivos, por socialização e equilíbrio emocional; conversação e desenvoltura social, pelo conjunto equilibrado de todas os cinco grandes fatores, exceto escrupulosidade; auto-exposição a desconhecidos e a situações novas, por extroversão e equilíbrio emocional; e auto-controle da agressividade por socialização.

Palavras-chave: Social skills; personality traits; Modelo dos cinco grandes fatores.
\end{abstract}

\section{A correlational study of social skills to personality traits}

\begin{abstract}
The purpose of this study was to investigate de correlation of social skills to personality traits by the big five factors model. The Ss were 189 undergraduates, 41 men and 148 women, from 18 to 59 ys. old (mean 26,3 ys. old), freshmen of the Literature, History, Psychology, Pedagogy, and Biology courses. The instruments were the Social Skills Inventory, which informs about (a) confront with risk, (b) self-affirmation on the positive affect expression, (c) conversation and social easiness, (d) self-exposure to strangers and new situations, and (e) self-control of aggressiveness in aversive situations; and an Personality Inventory which informs about the big five factors: extraversion, agreeableness, conscientiousness, neuroticism and openness. The confront with risk variance was explained by the extraversion and openness; self-affirmation on the positive affect expression, by agreeableness and emotional balance; conversation and social nimbleness, by the equality in all personality factors; self-exposure to strangers and new situations, by extraversion and emotional balance, and self-control of aggressiveness in aversive situations, by agreeableness.
\end{abstract}

Keywords: Habilidades social; traços de personalidade; Big Five Factors model.

\section{Introdução}

As habilidades sociais se constituíram como uma área dentro da Psicologia, na década de 60, na Inglaterra, tomando por base estudos relacionados à Psicologia Social e do Trabalho (Argyle \& Kendon, 1967) que disseminaram o termo habilidades sociais. Contudo, as décadas de 60 e 70 são marcadas por estudos relacionados à assertividade e ao treinamento assertivo, uma área tida por muitos como precursora do que viria a se desenvolver a partir da década de 80 e firmar-se na de 90 como treinamento de habilidades sociais (THS), um campo de investigação e de aplicação do conhecimento psicológico sobre o desempenho social.

O treinamento assertivo se constitui num método para tratar ansiedade e facilitar "a expressão adequada de qualquer emoção que não a ansiedade em relação a outra pessoa" (Wolpe, 1976, p.98). Foi criticado, no entanto,

${ }^{1}$ Endereço para correspondência:

Rua Alberto Santos Dumont, 89 - Vila Mutton, Itatiba - SP, 13.251 -280.

E-mail: jmhbueno@uol.com.br por enfatizar a expressão de sentimentos negativos, argumentando-se que muitos sentimentos positivos também poderiam encontrar-se bloqueados neuroticamente, necessitando de expressão emocional (Lazarus, 1977). Por outro lado, o treinamento de habilidades sociais (THS) se constitui numa abordagem mais ampla que o treinamento assertivo, por aplicar-se a qualquer dificuldade de natureza interpessoal, como habilidades de comunicação, resolução de problemas, de cooperação, enquanto que $\mathrm{O}$ segundo tem se restringido às dificuldades de expressão de sentimentos e afirmação de direitos em função da ansiedade.

Vários trabalhos publicados relatam a utilização do treinamento de habilidades sociais com sucesso no tratamento de diversos tipos de pacientes psiquiátricos (Argyle \& Trower, 1974; Rice \& Chaplin, 1979; Spencer, Gillespie \& Ekisa, 1983; Wallace \& Liberman, 1985; Schreuder, 1987), alcoolistas (Oei \& Jackson, 1980), com desordem de personalidade evitativa (Stravisnky, Grey \& Elie, 1987; Stravisnky, Lesage, Marcouiller \& Elie, 1989) e depressão (Jackson, Moss \& Solinski, 1985); além de 
programas para o desenvolvimento da auto-estima de crianças (Bockoven \& Morse, 1986), e na reeducação social com presidiários (Valliant \& Antonowicz, 1991).

Outros trabalhos investigam a relação das habilidades sociais com diversos critérios do mundo real. Pesquisas com crianças, por exemplo, mostraram que aquelas que possuem baixos níveis de compreensão interpessoal e auto-percepção de competência tendem a ser percebidas como problemáticas por seus pais (Kurdek \& Krile, 1983), e que as habilidades sociais têm grande importância na adaptação e no desempenho escolar (Togasaki \& Sakano, 1997; Carlyon, 1997).

Nos estudos com adultos, relatam-se várias diferenças nas habilidades sociais entre gêneros, como por exemplo, que as mulheres apresentam níveis de sociabilidade mais altos que os homens, e tendem a ser percebidas como mais atrativas fisicamente (Hidalgo et al, 1999; Margalit \& Eysenck, 1990; Saranson, Saranson, Hacker \& Basham, 1985). Além disso, várias características de personalidade aparecem envolvidas nas habilidades sociais. As desordens de personalidade tendem a comprometê-las (Penn e cols., 1999; Petti, 1999), enquanto que as características positivas, como auto-estima, tendem a estar presentes em sujeitos mais expressivos e com tato social, fazendo com que tenham mais credibilidade com os outros do que os sujeitos com ansiedade-social (Riggio, Throckmorton \& DePaola, 1990; Riggio, Tucker \& Throckmorton, 1987). De uma forma geral, foi encontrado que a competência social é capaz de prever o nível de felicidade que o indivíduo encontrará na vida (Argyle \& Lu, 1990).

No Brasil, poucos trabalhos foram realizados nessa área e se concentram principalmente no estudo das habilidades sociais de estudantes de Psicologia ( Del Prete, Del Prete \& Barreto, 1999; Del Prete, Del Prete \& Correia,1992), de professores (Del Prette, Del Prette, Garcia, Silva \& Puntel, 1998) e de menores de rua (Campos, Del Prette \& Del Prette, 2000). Além disso, um instrumento, o Inventário de Habilidades Sociais (Del Prette, Del Prette e Barreto, 1998), utilizado neste trabalho, foi desenvolvido para avaliar cinco fatores das habilidades sociais. O Fator 1, enfrentamento com risco, retrata situações interpessoais de afirmação de direitos e de auto-estima, mesmo diante do risco potencial de reação indesejável por parte do interlocutor. Expressa o conceito de assertividade. $\mathrm{O}$ Fator 2, auto-afirmação na expressão de afetos positivos, reúne itens em que ocorre a expressão de afeto positivo e afirmação da auto-estima, com risco mínimo de reação indesejável por parte do interlocutor. A conversação e desenvoltura social, expressa pelo Fator 3, envolve situações sociais neutras, com risco mínimo de reação indesejável, demandando conhecimento das normas sociais de relacionamento cotidiano para conversação. Situações que envolvem a abordagem a pessoas desconhecidas constituem a autoexposição a desconhecidos on a situaçôes novas, abordadas no Fator 4. Finalmente, no Fator 5, autocontrole da agressividade a situacõos aversivas, refere-se ao controle da raiva e da agressividade em situações de estimulações aversivas por parte do interlocutor.

Supõe-se que tais habilidades sociais e traços de personalidade se influenciem mutuamente, isto é, traços específicos de personalidade devem facilitar determinadas formas de expressão social e dificultar outras, assim como a forma peculiar do sujeito se comportar socialmente, as gratificações e frustrações que obtém a partir do contato com outras pessoas, também devem influenciar a organização de sua personalidade. A compreensão das concomitâncias peculiares existentes entre essas duas variáveis poderia ser de grande valia em programas de seleção, desenvolvimento, treinamento e aconselhamento psicológicos. O modelo dos cinco grandes fatores de personalidade, por ser bastante simples e abrangente, pareceu-nos o mais adequado para explorar relações entre traços de personalidade $\mathrm{e}$ habilidades sociais.

Esse modelo surgiu empiricamente, com base em análises de instrumentos tradicionais (como o 16PF, o MMPI, as escalas de personalidade de Comrey, entre outros) isolados ou em conjunto, cujas soluções fatoriais apontavam sempre para os mesmos cinco fatores, independentemente da teoria em que seus respectivos autores se basearam para desenvolvê-los. Só a partir de então é que começaram a surgir instrumentos especialmente construídos para avaliação da personalidade dentro do modelo dos cinco grandes fatores. O NEO-PI-R (Costa \& McCrae, 1992), pode ser citado como o principal instrumento utilizado para essa medida no mundo e, no Brasil, Hutz e colaboradores (1998) identificaram 64 marcadores de traços com bons resultados de fidedignidade e validade fatorial.

O Modelo dos Cinco Grandes Fatores descreve os seguintes traços de personalidade: altos escores em extroversão revelam pessoas sociáveis, ativas, falantes, otimistas e afetuosas; enquanto que baixos escores revelam pessoas que tendem a ser reservadas, sóbrias, indiferentes, independentes e quietas; o fator socialização descreve pessoas que tendem a ser generosas, bondosas, afáveis, prestativas e altruísticas de um lado e pessoas cínicas, não cooperativas e irritáveis, podendo chegar a ser manipuladoras, vingativas e implacáveis, de outro; alta pontuação em escrupulosidade indica comportamentos no sentido da organização, persistência, controle e motivação para alcançar objetivos altruísticos; enquanto que baixas pontuações revelam pessoas que tendem a não ter objetivos claros, não serem confiáveis e serem 
preguiçosas, negligentes e hedonistas; no fator neuroticismo, indivíduos com escores altos são propensos a sofrimentos psicológicos, podendo apresentar idéias irreais, baixa tolerância à frustração e respostas de coping não adaptativas, enquanto que indivíduos mais equilibrados emocionalmente, apresentam escores mais baixos nesse fator; altos escores no fator Abertura para novas experiências são obtidos por indivíduos curiosos, imaginativos e criativos, que se divertem com novas idéias e valores não convencionais, enquanto que baixos escores são obtidos por indivíduos que tendem a ser convencionais em suas crenças e atitudes, conservadores em suas preferências e menos responsivos emocionalmente.

O objetivo deste trabalho, portanto, é a investigação das correlações existentes entre habilidades sociais e traços de personalidade, partindo-se da hipótese de que as diferentes habilidades sociais podem ser explicadas por diferentes traços de personalidade.

\section{Método}

\section{Sujeitos:}

Foram sujeitos da pesquisa 189 estudantes universitários, de ambos os sexos (41 homens e 148 mulheres), de 18 a 59 anos $(M=26,3$ anos; $\mathrm{DP}=9,30)$, primeiro-anistas dos cursos de Letras, História, Psicologia, Pedagogia e Biologia.

\section{Instrumentos, aplicação e correção}

O instrumento para avaliação dos Cinco Grandes Fatores de Personalidade é uma escala de 64 adjetivos (Hutz e cols., 1998) na qual cada sujeito atribui pontos de 1 a 5 , conforme considere que o adjetivo não o descreve adequadamente até que o descreve adequadamente. As pontuações dos fatores podem variar nos seguintes intervalos: extroversão, de 10 a $50(\mathrm{M}=34,87$; $\mathrm{DP}=8,88)$; socialização, de 16 a $80(\mathrm{M}=64,88$; $\mathrm{DP}=10,28)$; escrupulosidade, de 14 a $70(\mathrm{M}=53,73 ; \mathrm{DP}=7,63)$; neuroticismo, de 12 a $60(\mathrm{M}=28,35$; $\mathrm{DP}=6,76)$; e abertura para novas experiências, de 0 a $12(\mathrm{M}=40,21 ; \mathrm{DP}=7,80)$.

Como medida dos cinco fatores de habilidades sociais, foi utilizado o Inventário de Habilidades Sociais (Del, Prette, Del Prette \& Barreto, 1998), composto de 31 itens. O sujeito atribui pontos de 0 a 4, conforme considere os comportamentos descritos como nunca ou raramente presentes até sempre ou quase sempre presentes, respectivamente. A pontuação do fator enfrentamento com risco pode variar de 0 a 44 $(M=23,08 ; \mathrm{DP}=7,55)$; da auto-afirmação na expressão de afeto positivo, de 0 a $28(\mathrm{M}=19,25 ; \mathrm{DP}=3,89)$; da conversação e desenvoltura social, de 0 a $28(\mathrm{M}=17,73$; $\mathrm{DP}=4,43)$; da auto-exposição a desconhecidos ou a situações novas, de 0 a $12(\mathrm{M}=7,41 ; \mathrm{DP}=2,66)$; e da auto-controle da agressividade a situações aversivas, de 0 a $12(\mathrm{M}=6,92 ; \mathrm{DP}=1,88)$.

Ambos os inventários foram aplicados coletivamente, em sala de aula, seguindo os padrões éticos. Os escores em cada habilidade social e traço de personalidade, foram obtidos somando-se as pontuações atribuídas pelos sujeitos aos itens que compõem cada fator.

\section{Resultados}

Inicialmente, foram realizadas análises de variância e correlacionais das habilidades sociais. Encontraram-se diferenças estatisticamente significativas entre gêneros nas habilidades de enfrentamento com risco ( $\mathrm{F}=4,281$; $\mathrm{p}=0,040)$ e auto-afirmação na expressão de afetos positivos $(\mathrm{F}=5,078 ; \mathrm{p}=, 025)$. Para a primeira, encontrou-se média mais elevada para o sexo masculino $(\mathrm{M}=25,22 ; \mathrm{DP}=$ $7,40)$ do que para o feminino $(M=22,49$; $D P=7,51)$; e para a segunda, o inverso, média mais elevada para o sexo feminino $(M=19,58 ; \mathrm{DP}=3,84)$ do que para $\mathrm{O}$ masculino $(M=18,05 ; \mathrm{DP}=3,90)$. A análise correlacional entre habilidades sociais e idade não evidenciou qualquer resultado estatisticamente significativo, sugerindo que as habilidades sociais não se desenvolvem ou melhoram com a idade.

Foram realizadas outras análises correlacionais entre traços de personalidade de per si, habilidades sociais entre si e entre todas as variáveis. Os resultados são apresentados separadamente, em três conjuntos.

Tabela 1. Coeficientes de Correlação Pearson entre as Características de Personalidade

\begin{tabular}{|c|c|c|c|c|c|}
\hline & Abertura a exp. & Extroversão & Neuroticismo & Escrupulosidade & Socialização \\
\hline Abertura a exp. & $1,000(* *)$ & $0,253(* *)$ & $-0,132$ & $0,471(* *)$ & $0,394(* *)$ \\
\hline Extroversão & 0,253 (**) & $1,000(* *)$ & $-0,550(* *)$ & 0,060 & 0,109 \\
\hline Neuroticismo & $-0,132$ & $-0,550(* *)$ & $1,000(* *)$ & $-0,177(*)$ & $-0,191(* *)$ \\
\hline Escrupulosidade & 0,471 (**) & 0,060 & $-0,177(*)$ & $1,000(* *)$ & $0,548(* *)$ \\
\hline Socialização & $0,394(* *)$ & 0,109 & $-0,191(* *)$ & $0,548(* *)$ & $1,000(* *)$ \\
\hline
\end{tabular}

** Correlações significativas no nível de 0.01 (2-tailed).

*Correlações significativas no nível de 0.05 (2-tailed). 
Como pode ser observado na Tabela 1 , extroversão apresentou alta correlação negativa com neuroticismo e positiva com abertura para novas experiências, socialização e escrupulosidade, além de apresentarem altas correlações positivas entre si, também se correlacionaram positivamente com abertura para novas experiências e negativamente com neuroticismo; este último não apresentou correlação estatisticamente significativa apenas com abertura para novas experiencias que, por sua vez, apresentou altas correlações positivas com escrupulosidade, socialização e extroversão.

A Tabela 2 apresenta as correlações entre os componentes das habilidades sociais:

Tabela 2. Coeficientes de Correlação Pearson entre os fatores de Habilidades Sociais

\begin{tabular}{|c|c|c|c|c|c|}
\hline & $\begin{array}{c}\text { Enfrentamento } \\
\text { com risco } \\
(\text { HS 1) } \\
\end{array}$ & $\begin{array}{l}\text { Auto-afirmação na } \\
\text { expressão de } \\
\text { afetos positivos } \\
\text { (HS 2) }\end{array}$ & $\begin{array}{c}\text { Conversação e } \\
\text { desenvoltura } \\
\text { social } \\
\text { (HS 3) } \\
\end{array}$ & $\begin{array}{c}\text { Auto-exposição a } \\
\text { desconhecidos ou a } \\
\text { situações novas } \\
\text { (HS 4) }\end{array}$ & $\begin{array}{c}\text { Auto-controle da } \\
\text { agressividade a } \\
\text { situações aversivas } \\
\text { (HS 5) } \\
\end{array}$ \\
\hline HS1 & $1.000(* *)$ & $0.418(* *)$ & $0.193(* *)$ & $0.172(*)$ & -0.075 \\
\hline HS2 & $0.418\left(^{* *}\right)$ & $1.000(* *)$ & 0.091 & 0.084 & 0.041 \\
\hline HS3 & 0.193 (**) $^{* *}$ & 0.091 & $1.000(* *)$ & $0.332(* *)$ & 0.144 \\
\hline HS4 & 0.172 & 0.084 & $0.332(* *)$ & $1.000(* *)$ & 0.032 \\
\hline HS5 & -0.075 & 0.041 & $0.144\left(^{*}\right)$ & 0.032 & $1.000(* *)$ \\
\hline
\end{tabular}

** Correlações significativas no nível de 0.01 (2-tailed).

* Correlações significativas no nível de 0.05 (2-tailed).

A Tabela 2 mostra que o enfrentamento com risco apresentou-se altamente correlacionado com autoafirmação na expressão de afetos positivos e correlações mais baixas, mas também estatisticamente significativas, com conversação e desenvoltura social e auto-exposição a desconhecidos ou a situações novas. A auto-afirmação na expressão de afetos positivos não se correlacionou significativamente com qualquer outra habilidade social, exceto com a anterior, podendo-se entender que o enfrentamento com risco compreende a maior parte das habilidades da autoafirmação na expressão de afetos positivos, mas não o contrário.
O fator conversação e desenvoltura social correlacionou-se com as habilidades de auto-exposição a desconbecidos on a situaçôes novas e auto-controle da agressividade a situacões aversivas, demandando tanto a capacidade de se expor, quanto o controle da agressividade. $\mathrm{O}$ auto-controle da agressividade a situaģẽes aversivas correlacionou-se apenas com a conversação e desenvoltura social, e em nível muito baixo.

Por fim, a Tabela 3, com as correlações entre os componentes das habilidades sociais e traços de personalidade completam os 3 conjuntos de análises anteriormente referidos.

Tabela 3. Correlações entre Habilidades Sociais e Características de Personalidade

\begin{tabular}{|c|c|c|c|c|}
\hline $\begin{array}{c}\text { Enfrentamento } \\
\text { com risco }\end{array}$ & $\begin{array}{l}\text { Auto-afirmação } \\
\text { na expressão de } \\
\text { afetos positivos }\end{array}$ & $\begin{array}{c}\text { Conversação e } \\
\text { desenvoltura } \\
\text { social }\end{array}$ & $\begin{array}{l}\text { Auto-exposição a } \\
\text { desconhecidos ou } \\
\text { a situações novas }\end{array}$ & $\begin{array}{l}\text { Auto-controle da } \\
\text { agressividade a } \\
\text { situações aversivas }\end{array}$ \\
\hline $0,552(* *)$ & $0,209(* *)$ & $0,304(* *)$ & ,301 (**) & $-0,004$ \\
\hline $0,187(* *)$ & $0,393(* *)$ & $0,240(* *)$ & 0,033 & $0,216(* *)$ \\
\hline $0,172(*)$ & $0,268(* *)$ & 0,207 (**) & $-0,019$ & $0,168\left(^{*}\right)$ \\
\hline$-0,270(* *)$ & $-0,270(* *)$ & $-0,314(* *)$ & $-0,311(* *)$ & $-0,161\left(^{*}\right)$ \\
\hline $0,395(* *)$ & 0,239 (**) & $0,250(* *)$ & 0,090 & $-0,007$ \\
\hline
\end{tabular}

** Correlações significativas no nível de 0.01 (2-tailed).

* Correlações significativas no nível de 0.05 (2-tailed).

Pode-se observar uma grande quantidade de correlações significativas entre as variáveis: as três primeiras habilidades sociais, enfrentamento com risco, auto-afirmação na expressão de afetos positivos e conversação e desenvoltura social apresentaram correlações estatisticamente significativas com todos os traços de personalidade; auto-exposição a desconbecidos on a situacões novas correlacionou-se significativamente apenas com extroversão e neuroticismo; e auto- controle da agressividade a situações aversivas correlacionou-se significativamente com socialização e neuroticismo. Como alguns traços de personalidade também apresentaram correlações significativas entre si, decidiu-se por proceder a correlações parciais entre as variáveis, controlando os traços de personalidade.

A tabela 4 mostra as correlações parciais entre enfrentamento com risco e traços de personalidade. 
Tabela 4 - Coeficientes de Correlação parcial de Pearson entre enfrentamento com risco e traços de personalidade.

\begin{tabular}{|c|c|c|c|c|c|}
\hline & \multicolumn{5}{|l|}{ Controle por } \\
\hline & Extrov. & Social. & Escrup. & Neurot. & Abert. Exp. \\
\hline Extroversão & & $0,5448(* *)$ & $0,5512 \quad(* *)$ & $0,5022(* *)$ & $0,5090(* *)$ \\
\hline Socialização & $0,1529(*)$ & & 0,1121 & $0,1430(*)$ & 0,0368 \\
\hline Escrupulosidade & $0,1673(*)$ & 0,0851 & & 0,1313 & $-0,0171$ \\
\hline Neuroticismo & 0,0484 & $-0,2430(* *)$ & $-0,2470(* *)$ & & $-0,2391(* *)$ \\
\hline Abertura $\mathrm{p} /$ novas experiências & $0,3166(* *)$ & $0,3561(* *)$ & $0,3613(* *)$ & $0,3766(* *)$ & \\
\hline
\end{tabular}

Quando as correlações com a habilidade de enfrentamento com risco foram controladas por cada traço de personalidade, os únicos que continuam apresentando correlações estatisticamente significativas foram extroversão e abertura para novas experiências (Tabela 4). Socialização e escrupulosidade perderam significância estatística quando controladas uma pela outra, ou ambas por abertura para novas experiências; escrupulosidade também perdeu significância quando controlada por neuroticismo; e este último perdeu significância quando controlado por extroversão.

A tabela 5, por sua vez, mostra as correlações parciais entre auto-afirmação na expressão de afetos positivos e traços de personalidade.

Tabela 5. Coeficientes de Correlação parcial entre auto-afirmação na expressão de afetos positivos e traços de personalidade.

\begin{tabular}{|c|c|c|c|c|c|}
\hline \multirow[t]{2}{*}{ 年 } & \multicolumn{5}{|l|}{ Controle por } \\
\hline & Extrov. & Social. & Escrup. & Neurot. & Abert. Exp. \\
\hline Extroversão & & $0,1816(*)$ & $0,1816^{(* *)}$ & 0,0751 & $0,1577(*)$ \\
\hline Socialização & $0,3808(* *)$ & & $0,0689(* *)$ & $0,3611(* *)$ & $0,3346(* *)$ \\
\hline Escrupulosidade & $0,2620(* *)$ & 0,0689 & & $0,2326(* *)$ & $0,1817(*)$ \\
\hline Neuroticismo & $-0,1897(* *)$ & $-0,1897(* *)$ & $-0,2156(* *)$ & & $-0,2474(* *)$ \\
\hline Abertura $\mathrm{p} /$ novas experiências & $0,1968(* *)$ & 0,1968 & 0,0996 & $0,2130(* *)$ & \\
\hline
\end{tabular}

** Correlações significativas no nível de 0.01 (2-tailed).

* Correlações significativas no nível de 0.05 (2-tailed).

O fator auto-afirmação na expressão de afetos positivos manteve correlações estatisticamente significativas com os traços de socialização e neuroticismo, mesmo quando essas correlações foram controladas por outras traços de personalidade (Tabela 5). Extroversão perdeu significância quando controlada por neuroticismo; escrupulosidade, quando controlada por socialização; e abertura para novas experiências, quando controlada por socialização ou escrupulosidade.

Os dados da conversação e desenvoltura social correlacionados parcialmente com traços de personalidade são apresentados na tabela 6 .

Tabela 6. Coeficientes de Correlação parcial entre conversação e desenvoltura social com traços de personalidade.

\begin{tabular}{|c|c|c|c|c|c|}
\hline & \multicolumn{5}{|l|}{ Controle por } \\
\hline & Extrov. & Social. & Escrup. & Neurot. & Abert. Exp. \\
\hline Extroversão & & $0,2880(* *)$ & $0,2986(* *)$ & $0,1658\left(^{*}\right)$ & $-0,2569(* *)$ \\
\hline Socialização & $0,2189(* *)$ & & $0,1552(*)$ & $0,1936(* *)$ & $-0,1592(*)$ \\
\hline Escrupulosidade & $0,1984(* *)$ & 0,0926 & & $0,1619(*)$ & $-0,1041$ \\
\hline Neuroticismo & $-0,1841(*)$ & $-0,2810(* *)$ & $-0,2877(* *)$ & & $0,2923(* *)$ \\
\hline Abertura p/ novas experiências & $0,1882(* *)$ & $0,1745(*)$ & $0,1772(*)$ & $0,2220(* *)$ & \\
\hline
\end{tabular}

A correlação entre a habilidade de conversação e desenvoltura social e o traço de escrupulosidade, perdeu significância estatística quando controlada por socializaçãa ou abertura para novas experiências; os demais traços de personalidade mantiveram correlações estatisticamente Psico-USF, v.6, n.1, p.31-38, jan./jun. 2001 significativas mesmo quando controlados uns pelos outros (Tabela 6). A conversação e desenvoltura social, assim, requer um conjunto de traços de personalidade, porém não necessariamente altruísticos. 
A auto-exposição a desconhecidos on a situaçoes novas manteve correlações estatisticamente significativas com os traços de extroversão $(\mathrm{r}=0,1630 ; \mathrm{p}=0,025)$ e neuroticismo $(\mathrm{r}=-0,1833, \mathrm{p}=0,012)$, mesmo quando os traços de personalidade são controlados um pelo outro.
$\mathrm{Na}$ tabela 7 são apresentadas as correlações parciais entre auto-controle da agressividade a situações aversivas e traços de personalidade.

Tabela 7. Correlações parciais de o auto-controle da agressividade a situações aversivas.

\begin{tabular}{lllll}
\cline { 3 - 4 } & \multicolumn{3}{c}{ Controle por } & \\
\cline { 2 - 5 } & Socialização & Escrupulosidade & Neuroticismo \\
\hline Socialização & r & &, $1507\left(^{*}\right)$ & $0,1914(* *)$ \\
\hline Escrupulosidade & r & $-0,0603$ & & $0,1434(*)$ \\
\hline Neuroticismo & r & 0,1244 &,- 1349 & \\
\hline
\end{tabular}

** Correlações significativas no nível de 0.01 (2-tailed).

* Correlações significativas no nível de 0.05 (2-tailed).

$\mathrm{O}$ auto-controle da agressividade a situações aversivas, que inicialmente mostrou-se correlacionado com socialização, escrupulosidade e neuroticismo, manteve correlação significativa apenas com socialização quando os traços de personalidade iniciais foram controlados (Tabela 7).

\section{Conclusão}

Este estudo analisou as relações existentes entre habilidades sociais e traços de personalidade (pelo modelo dos cinco grandes fatores), encontrando evidências que apóiam pesquisas anteriores em relação à diferenças entre gêneros nas duas primeiras habilidades sociais (Hidalgo et al, 1999; Margalit \& Eysenck, 1990; Saranson, Saranson, Hacker \& Basham, 1985) e, como previsto, a associação de diferentes traços de personalidade à diferentes habilidades sociais. $\mathrm{O}$ enfrentamento com risco correlacionou-se positivamente com extroversão e abertura para novas experiências; a auto-afirmação na expressão de afetos positivos correlacionou-se positivamente com socialização e equilíbrio emocional; a conversação e desenvoltura social mostrou-se relacionada a todos os traços de personalidade, exceto a escrupulosidade; auto-exposição a desconhecidos ou a situações novas correlacionou-se positivamente com extroversão e equilibrio emocional; e auto-controle da agressividade a situações aversivas correlacionou-se positivamente com socialização.

Com base nesses resultados pode-se interpretar algumas relações de interesse para compreensão do psiquismo humano. Pessoas que relataram enfrentar situações com risco de reação indesejável pelo interlocutor, também se descreveram como mais extrovertidas e abertas a novas experiências no inventário de personalidade, parecendo unir facilidade/desejo de exposição (extroversão) com a confiança necessária para readaptação (abertura para novas experiências), caso a reação indesejável implique em mudanças mais profundas. Situações de auto-afirmação na expressão de afetos positivos, com risco mínimo de reação indesejável pelo interlocutor, foram relatadas por pessoas com altos escores em socialização e baixos em neuroticismo, capazes de criar uma impressão favorável de si mesmas, conquistando o interlocutor e evitando áreas de conflito. A conversação e desenvoltura social foi a habilidade que se correlacionou com o maior número de traços de personalidade, mostrando-se a mais complexa entre as habilidades sociais analisadas. De fato, é necessário um conjunto equilibrado de traços de personalidade para iniciar uma conversação, mantê-la interessante e ainda dentro das exigências sociais, porém, não necessariamente este seja um contato altruístico, já que a escrupulosidade foi o único traço que perdeu significância estatística quando controlado por outros traços de personalidade. A auto-exposição a desconhecidos ou a situações novas é característica de pessoas extrovertidas e equilibradas emocionalmente (não neuróticas). Capazes de acreditar em si mesmas, essas pessoas acreditam que serão bem aceitas, ou, no caso não o serem, que pelo menos serão capazes de lidar com adversidades próprias do convívio humano. O auto-controle da agressividade requer principalmente o interesse pelo bem-estar social, conferido pelo traço de socialização; provavelmente, em seu desenvolvimento, tais pessoas tenham aprendido a reconhecer as necessidades de outras pessoas e a priorizar o bem-estar do grupo, muitas vezes, em detrimento do seu próprio.

Curiosamente, o traço de escrupulosidade, que inicialmente se correlacionou com todas as habilidades sociais, exceto com HS4 (auto-exposição a desconhecidos ou a situações novas), não manteve correlações significativas com qualquer habilidade social, quando as correlações foram controladas por outros traços de personalidade. Este, talvez, seja o ponto que mereça 
outras investigações, pois a escrupulosidade é o traço que garantiria o sentido altruístico das relações sociais e esse resultado indica que habilidades sociais e altruísmo não necessariamente andam juntos. Há que se ressalvar, porém, o fato de escrupulosidade ter apresentado alta correlação com socialização e abertura para novas experiências, podendo o caráter altruístico das relações sociais ter sido expresso indiretamente pela socialização, já que abertura para novas experiências parece estar mais ligada à capacidade de realização das pessoas no traço de escrupulosidade.

Pelos resultados encontrados, este trabalho também apresenta evidências de validade concorrente entre os dois instrumentos. Amplia, em conseqüência, suas utilizações em programas de seleção, desenvolvimento, treinamento e orientação profissional, aconselhamento psicológico, etc., possibilitando interpretações cruzadas das habilidades dos sujeitos e maior abrangência para uma análise psicológica.

\section{Referências Bibliográficas}

Argyle, M. \& Kendon, A. (1967). The experimental analysis of social performance, Advances in Experimental Social Psychology, 3, p. 55-98.

Argyle, M.; Lu, L. (1990). Happiness and social skills. Personality and Individual Differences, vol. 11(12): 1255-1261.

Argyle, M.; Trower, P. (1974). Explorations in the treatment of personality disorders and neuroses by social skills training. British Journal of Medical Psychology, Vol. 47(1): 6372.

Bockoven, J.; Morse, C. L. (1986). A comparative study of the efficacy of the DUSO and DUSO-R on children's social skills and self-esteem. Elementary School Guidance and Counseling, Vol. 20(4): 290-296.

Carlyon, W. D. (1997). Attribution retraining: Implications for its integration into prescriptive social skills training. School Psychology Review, Vol. 26(1): 61-73.

Campos, T. N.; Del Prette, Z. A. P. \& Del Prette, A. (2000). (Sobre)vivendo nas ruas: habilidades sociais e valores de crianças e adolescentes. Psicologia: Reflexão e Crítica, vol.13 no.3.

Costa, P. T. Jr., \& McCrae, R. R. (1992). Prevised NEO Personality Inventory (NEO-PI-R) and NEO Five-Factor Inventory (NEO-FFI) professional manual. Odessa, FL: Psychological Assessment Resources.

Del Prette, A.; Del Prette, Z. A. P. \& Barreto, M. C. M. (1999). Habilidades sociales en la formación del psicólogo: analisis un programa de intervención. Psicología Concuctual, 7(1), 2747.

Del Prette, A.; Del Prette, Z. A. P. \& Barreto, M. C. M. (1998). Análise de um Inventário de Habilidades Sociais (IHS) em uma amostra de universitários. Psicologia: Teoria e Pesquisa, vol.14, no.3, pp. 219-228.

Del Prette, Z. A. P.; Del Prette, A. \& Correia, M. F. B. (1992). Competência Social: Um estudo comparativo entre alunos de Psicologia, Serviço Social e Engenharia Mecânica. Psicólogo Escolar: Identidade e Perspectivas, p. 282384.

Del Prette, Z. A. P.; Del Prette, A.; Garcia, F. A.; Silva, A. T. B. \& Puntel, L. P. (1998). Habilidades sociais do professor em sala de aula: um estudo de caso. Psicologia: Reflexão e Crítica, vol.11, no.3.

Hidalgo Montesinos, M. D.; Galindo Garre, F.; Ingles Saura, C. J.; Campoy Menendez, G.; Ortiz Soria, B. (1999). A study of differential item functioning in a social skills scale for adolescents. Anales de Psicologia; Vol. 15(2): 331343.

Hutz, C. S.; Nunes, C. H. S. S.; Serra, J.; Silveira, A. D. \& Anton, M. (1998). O desenvolvimento de marcadores para a avaliação da personalidade no modelo dos cinco grandes fatores. Psicologia : Reflexão e Crítica, 11, 395-410.

Jackson, H. J.; Moss, J. D.; Solinski, S. (1985). Social skills training: An effective treatment for unipolar nonpsychotic depression? Australian-and New Zealand Journal of Psychiatry, Vol. 19(4): 342-353.

Kurdek, L. A.; Krile, D. (1983). The relation between thirdthrough eighth-grade children's social cognition and parents' ratings of social skills and general adjustment. Journal of Genetic Psychology, Vol. 143(2): 201-206.

Lazarus, A. A. (1977). Psicoterapia Personalista - Uma visão além do condicionamento. Belo Horizonte: Interlivros.

Margalit, M.; Eysenck, S. (1990). Prediction of coherence in adolescence: Gender differences in social skills, personality, and family climate. Journal of Research in Personality. Vol. 24(4): 510-521

Oei, T. P.; Jackson, P. (1980). Long-term effects of group and individual social skills training with alcoholics. Addictive Behaviors. Vol. 5(2): 129-136.

Penn, D. L.; Corrigan, P. W.; Martin, J.; Ihnen, G.; Racenstein, J. Meg.; Nelson, D.; Cassisi, J.; Hope, D. A. (1999). Social cognition and social skills in schizophrenia: The role of self-monitoring. Journal of Nervous and Mental Disease. Vol. 187(3): 188-190.

Petti, V. L. (1999). Emotion perception competence and its relationship to social skills, personality characteristics, and self-concept of children with varied cognitive abilities in a psychiatric sample. Dissertation Abstracts International: Section B: The Sciences and Engineering. Vol. 59(8-B): 4480 .

Rice, M. E.; Chaplin, T. C. (1979). Social skills training for hospitalized male arsonists. Journal of Behavior Therapy and Experimental Psychiatry. Vol. 10(2): 105-108.

Riggio, R. E.; Throckmorton, B.; DePaola, S. (1990). Social skills and self-esteem. Personality and Individual Differences. Vol. 11(8): 799-804

Riggio, R. E.; Tucker, J.; Throckmorton, B. (1987). Social skills and deception ability. Personality and Social Psychology Bulletin. Vol. 13(4): 568-577.

Saranson, B. R.; Saranson, I. G.; Hacker, T. A.; Basham, R.B. (1985). Concomitants of social support: Social skills, physical attractiveness, and gender. Journal of Personality and Social Psychology. Vol. 49(2): 469-480.

Schreuder, M. (1987). Social skills training in psychiatric 
outpatients. Gedragstherapie. Vol. 20(4): 271-283.

Spencer, P. G.; Gillespie, C. R.; Ekisa, E.G. (1983). A controlled comparison of the effects of social skills training and remedial drama on the conversational skills of chronic schizophrenic inpatients. British Journal of Psychiatry. Vol. 143: 165-172.

Stravynski, A; Grey, S.; Elie, R. (1987). Outline of the therapeutic process in social skills training with socially dysfunctional patients. Journal of Consulting and Clinical Psychology. Vol. 55(2): 224-228.

Stravynski, A.; Lesage, A.; Marcouiller, M.; Elie, R. (1989). A test of the therapeutic mechanism in social skills training with avoidant personality disorder. Journal of Nervous and Mental Disease. Vol. 177(12): 739-744.

Togasaki, Y.; Sakano, Y (1997). Effects of mother's attitude for child rearing on social skills and school adaptation in elementary school children: From the point of view of the attitude for child rearing of active refusal type. Japanese Journal of Educational Psychology. Vol. 45(2): 173-182.

Valliant, P. M.; Antonowicz, D. H. (1991). Cognitive behaviour therapy and social skills training improves personality and cognition in incarcerated offenders. Psychological Reports. Vol. 68(1): 27-33.

Wallace, C. J.; Liberman, R. P. (1985). Social skills training for patients with schizophrenia: A controlled clinical trial. Psychiatry Research. Vol. 15(3): 239-247.

Wolpe, J.S. (1976). A prática da terapia comportamental. São Paulo: Brasiliense.

Sobre os autores:

José Maurício Haas Bueno é Psicólogo e Professor do Curso de Psicologia na Universidade de Pouso Alegre (UNIPA) - MG.

Sandra Maria da Silva Sales Oliveira é Psicóloga e Professora do Curso de Psicologia na Universidade de Pouso Alegre (UNIPA) - MG.

José Carlos da Silva Oliveira é Psicólogo e Professor do Curso de Psicologia na Universidade de Pouso Alegre (UNIPA) - MG. 